\title{
THE RELATION BETWEEN THE FLOW OF NON-NEWTONIAN FLUIDS AND TURBULENT NEWTONIAN FLUIDS*
}

\author{
By R. S. RIVLIN (Brown University)
}

It is well known [1] $]^{* *}$ that if a Newtonian fluid such as water flows through a tube of circular cross-section under conditions for which the critical Reynolds number is exceeded, the relation between pressure gradient and volume rate of flow is non-linear. Further [2], the radial distribution of velocity (averaged over regions large compared with the eddy dimensions) departs markedly from the parabolic distribution which would be expected for a Newtonian fluid. These results suggest that some analogy may exist between the turbulent Newtonian fluid and the laminar flow of a non-Newtonian fluid. It is the object of this note to pursue this line of thought and to indicate some of the phenomena which might, in accordance with it, be observed in turbulent fluids.

If we assume that in a fluid, which is isotropic in its state of rest, the stress components in the rectangular Cartesian coordinate system $x_{i}$ may be expressed as polynomials in the gradients of velocity, acceleration, second acceleration, $\cdots,(n-1)$ th acceleration, then the stress matrix $\mathbf{T}\left(=\left\|t_{i i}\right\|\right)$ may be expressed as a matrix polynomial in $n$ kinematic matrices $\mathbf{A}_{1}, \mathbf{A}_{2}, \cdots, \mathbf{A}_{n}$, defined by $[3,4]$

$$
\mathbf{A}_{1}=\left\|A_{i j}^{(1)}\right\|=\left\|\frac{\partial v_{i}}{\partial x_{i}}+\frac{\partial v_{i}}{\partial x_{i}}\right\|
$$

and

$$
\begin{array}{r}
\mathbf{A}_{r}=\left\|A_{i j}^{(r)}\right\|=\left\|\frac{\partial A_{i j}^{(r-1)}}{\partial t}+v_{l} \frac{\partial A_{i j}^{(r-1)}}{\partial x_{l}}+A_{i l}^{(r-1)} \frac{\partial v_{l}}{\partial x_{i}}+A_{i l}^{(r-1)} \frac{\partial v_{l}}{\partial x_{i}}\right\| \\
(r=2,3, \cdots, n)
\end{array}
$$

where $v_{i}$ is the velocity of the fluid. The coefficients in this matrix polynomial are polynomial invariants, under orthogonal transformations, of $\mathbf{A}_{1}, \mathbf{A}_{2}, \cdots, \mathbf{A}_{n}$. If $\mathbf{A}_{r}=0$ for $r>2$, then $\mathbf{T}$ may be expressed as a matrix polynomial in the two matrices $\mathbf{A}_{1}$ and $A_{2}$. It has been shown [5] that it may then be expressed in the form

$$
\begin{aligned}
\mathbf{T}=-p \mathbf{I}+\alpha_{1} \mathbf{A}_{1}+ & \alpha_{2} \mathbf{A}_{2}+\alpha_{3} \mathbf{A}_{1}^{2}+\alpha_{4} \mathbf{A}_{2}^{2}+\alpha_{5}\left(\mathbf{A}_{1} \mathbf{A}_{2}+\mathbf{A}_{2} \mathbf{A}_{1}\right) \\
& +\alpha_{6}\left(\mathbf{A}_{1}^{2} \mathbf{A}_{2}+\mathbf{A}_{2} \mathbf{A}_{1}^{2}\right)+\alpha_{7}\left(\mathbf{A}_{1} \mathbf{A}_{2}^{2}+\mathbf{A}_{2}^{2} \mathbf{A}_{1}\right)+\alpha_{8}\left(\mathbf{A}_{1}^{2} \mathbf{A}_{2}^{2}+\mathbf{A}_{2}^{2} \mathbf{A}_{1}^{2}\right),
\end{aligned}
$$

where $p$ is arbitrary if the velocity field is specified, $I$ is the unit matrix and the $\alpha$ 's are polynomials in $\operatorname{tr} \mathbf{A}_{1}^{2}, \operatorname{tr} \mathbf{A}_{2}^{2}, \operatorname{tr} \mathbf{A}_{1}^{3}, \operatorname{tr} \mathbf{A}_{2}^{3}, \operatorname{tr} \mathbf{A}_{1} \mathbf{A}_{2}, \operatorname{tr} \mathbf{A}_{1}^{2} \mathbf{A}_{2}, \operatorname{tr} \mathbf{A}_{1} \mathbf{A}_{2}^{2}, \operatorname{tr} \mathbf{A}_{1}^{2} \mathbf{A}_{2}^{2}$. In deriving this result it is assumed that the fluid is incompressible.

It can be shown [6] that the assumption that the stress components at a point of the fluid can be expressed as polynomials in the gradients of the velocity, acceleration, etc. at that point is, under rather general conditions, equivalent to an assumption that the stress in an element of the fluid at any instant of time depends on the velocity gradients in the element at that instant of time and at previous instants.

* Received June 25, 1956. The results presented in this note were obtained in the course of research sponsored by the Office of Ordnance Research, U. S. Army, under Contract No. DA-19-020-3487.

${ }^{* *}$ Numbers in square brackets refer to the Bibliography at the end of the paper. 
We shall now consider the results obtained on the basis of this theory for the flow of a fluid along a straight pipe of non-circular cross-section. If we assume that each particle of the fluid moves in a rectilinear path parallel to the length of the pipe (in the $x_{3}$-direction say), so that

$$
v_{1}=v_{2}=0 \text { and } v_{3}=v_{3}\left(x_{1}, x_{2}\right),
$$

then it is easily seen, from (1), that $\mathbf{A}_{r}=0(r>2)$, so that Eq. (2) is applicable. We now substitute the expressions (3) for the velocity components in (2) and then substitute the resulting expressions for the stress components in the equations of motion with zero body forces. The three equations so obtained may be regarded as differential equations for the determination of $p$ and $v_{3}$, subject to the boundary condition that $v_{3}=0$ on the wall of the pipe. It has been shown by Ericksen [7] that these equations are generally incompatible unless the pipe is circular. (Exceptions occur for particular forms of the expression (2) for the stress; for example, the form $T=-p I+\eta A_{1}$ with $\eta$ constant, which represents the Newtonian fluid, provides such a case.) This incompatibility implies that for a non-Newtonian fluid to which Eq. (2) applies, no state of rectilinear flow along a non-circular pipe is in general possible, unless body forces are applied to the fluid. Green and Rivlin [8] have considered the particular case of a nearly Newtonian fluid for which the stress is given by

$$
\mathbf{T}=-p \mathbf{I}+\eta \mathbf{A}_{1}+\epsilon\left(c+\operatorname{tr} \mathbf{A}_{1}^{2}\right) \mathbf{A}_{1}^{2},
$$

where $\eta$ and $c$ are constants and $\epsilon$ is small enough so that any departure from the flow field predicted for the Newtonian fluid, obtained by taking $\epsilon=0$ in (4), may be treated as a first order perturbation. They have found, for the case of a pipe with elliptical cross-section, a flow field in which secondary flows in the cross-sectional planes are superimposed on the rectilinear flow. Further calculations with somewhat similar results have been carried out by Langlois and Rivlin [9] using a more general form than (4) for the constitutive equation of the nearly Newtonian fluid.

It has been reported [10] that when a Newtonian fluid flows down a straight pipe of non-circular cross-section, under conditions for which the fluid has become fully turbulent, the flow is no longer rectilinear, but a secondary flow exists in the cross-sectional planes of a type similar to that calculated by Green and Rivlin and by Langlois and Rivlin. This fact further suggests that the turbulent Newtonian liquid may, for certain purposes, be regarded as a non-Newtonian fluid.

On the basis of the theory for the flow of non-Newtonian fluids described above, we can derive the existence of other phenomena. If the fluid is in a state of flow with uniform velocity gradient $K$, described by

$$
v_{1}=K x_{2}, \quad v_{2}=v_{3}=0,
$$

then, from (5), (1) and (2), $\mathbf{A}_{r}=0(r>2)$ and the stress components $t_{i i}$ at each point of the fluid are given by [11]

$$
\begin{aligned}
t_{11} & =-p+\alpha_{3} K^{2} \\
t_{22} & =-p+K^{2}\left[\left(2 \alpha_{2}+\alpha_{3}\right)+4\left(\alpha_{4}+\alpha_{6}\right) K^{2}+8 \alpha_{8} K^{4}\right], \\
t_{12} & =K\left[\alpha_{1}+2 \alpha_{5} K^{2}+4 \alpha_{7} K^{4}\right], \\
t_{33} & =-p, \quad t_{23}=t_{31}=0 .
\end{aligned}
$$


Unlike the situation that exists in a Newtonian fluid, the normal components of stress are not equivalent to a hydrostatic pressure. This results [11] in a number of phenomena which have been observed in non-Newtonian fluids and may be called normal stress effects.

For example, it was remarked by Garner and Nissan [12] that, for certain fluids, if a cylindrical rod is rotated in the fluid, the fluid rises up the rod. For a Newtonian fluid, the fluid surface would remain horizontal under conditions for which the centrifugal forces are negligible and be depressed slightly at the stirrer if centrifugal forces were not negligible. The rise at the stirrer which occurs with the non-Newtonian fluids implies that a radial distribution of normal surface thrusts would have to be exerted on the free surface of the non-Newtonian fluid in order to maintain in it a velocity field in which each particle moves with uniform velocity in a horizontal circular path about the axis of rotation.

Again, Weissenberg [13] contained certain non-Newtonian fluids between two coaxial cylindrical containers, the clearance between the bases of which was small compared with their diameters. The outer cylinder was rotated at a constant angular velocity, while the inner one, to which were attached a number of vertical tubes communicating with the fluid through small holes, was held stationary. He found that the fluid rose in the tubes to an extent depending on their distance from the axis of rotation, the rise being greatest at the axis and decreasing with increasing radial distance. In the region between the discs, each particle of the fluid moves substantially in a horizontal circular path about the axis of rotation with a uniform velocity depending on the radial and vertical positions of the particle. The rise of fluid in the tubes implies that, in order to maintain this state of flow in the non-Newtonian fluids, surface tractions must be exerted on the plane surfaces of the fluid mass directed normally into the fluid. For a Newtonian fluid, of course, only shearing tractions are required in order to maintain a similar flow*.

It can be shown that the terms in the expressions for the stress components, which give rise to the secondary flow in the problem of the flow of a non-Newtonian fluid down a non-circular pipe mentioned above, also give rise to normal stress effects of the types observed by Garner and Nissan and by Weissenberg and this raises the possibility that similar normal stress effects may also be observed in turbulent Newtonian fluids in analogous situations.

It has been noted that the fluids in which normal stress effects have been observed are visco-elastic and this suggests that turbulent Newtonian fluids may similarly show some elastic effects under suitable experimental conditions. It may be remarked that in high-polymer solutions, which exhibit both normal stress and visco-elastic effects, the origin of the phenomena probably lies in the preferential orientation and extension of the dissolved high-polymer molecules when the liquid is sheared. The eddies in a turbulent Newtonian fluid will presumably undergo preferential orientation when the turbulent fluid is sheared providing a possible mechanism for the effects in the turbulent fluid. Also, the eddies in the sheared volume elements of the turbulent fluid would presumably

*This is not strictly correct if the effect of centrifugal forces is taken into account, but centrifugal forces would cause the fluid to rise in the vertical tubes increasingly with increasing radial distance from the axis of rotation. Furthermore, the effect observed with non-Newtonian fluids may be quite pronounced under conditions of negligible centrifugal force. 
be distributed in an anisotropic manner and this would also provide a possible mechanism for the effects discussed.

It will, of course, be appreciated that the above remarks are of a qualitative nature and are presented as tentative suggestions rather than as positive predictions. Certainly it appears quite likely that a phenomenological theory of the type considered, in which the stress in an element of the turbulent fluid (large compared with the eddy dimensions) is supposed to depend only on the kinematic variables in that element or on the velocitygradient history of that element, will be entirely adequate as a complete description of the flow properties of the turbulent fluid, since eddies can diffuse from one point of the fluid to another.

\section{REFERENCES}

1. See, for example, H. Schlichting. Boundary layer theory, translated by J. Kestin. Pergamon Press, London, 1955, p. 400 et seq.

2. See, for example, H. Schlichting, loc. cit. p. 404 et seq.

3. J. G. Oldroyd, Proc. Roy. Soc. A 200, 523 (1950)

4. R. S. Rivlin and J. L. Ericksen, J. Ratl. Mech. \& Anal. 4, 323 (1955)

5. R. S. Rivlin, J. Ratl. Mech. \& Anal. 4, 681 (1955)

6. A. E. Green and R. S. Rivlin (pending publication)

7. J. L. Ericksen, Quart. Appl. Math. 14, 318 (1956)

8. A. E. Green and R. S. Rivlin, Quart. Appl. Math. 14, 299 (1956)

9. W. Langlois and R. S. Rivlin (pending publication)

10. H. Schlichting, loc. cit. p. 416

11. R. S. Rivlin, J. Ratl. Mech. \& Anal. 5, 179 (1956)

12. F: H. Garner and A. H. Nissan, Nature 158, 634 (1946)

13. K. Weissenberg, Nature 159, 310 (1947) 